\title{
The Plasmodium vivax Pv41 surface protein: Identification and characterization
}

\author{
Diana I. Angel ${ }^{\text {, }}$, Alvaro Mongui a , Javier Ardila ${ }^{\text {a }}$, Magnolia Vanegas ${ }^{\mathrm{a}, \mathrm{b}}$, Manuel A. Patarroyo ${ }^{\mathrm{a}, \mathrm{b}, *}$ \\ a Molecular Biology Department, Fundación Instituto de Inmunología de Colombia (FIDIC), Carrera 50\#26-20, Bogotá, Colombia \\ ${ }^{\mathrm{b}}$ Universidad del Rosario, Calle 63D\#24-31, Bogota, Colombia
}

\section{A R T I C L E I N F O}

\section{Article history:}

Received 17 October 2008

Available online 5 November 2008

\section{Keywords:}

Malaria

Plasmodium vivax

Pv41

Bioinformatics

Six-cys domain

Vaccine candidate

\begin{abstract}
A B S T R A C T
Recently, Plasmodium vivax has been related to nearly $81 \%$ of malaria cases reported in Central America and the Mediterranean. Due to the difficulty of culturing this parasite species in vitro, most studies on $P$. vivax have focused on the identification of new antigens by homology comparison with $P$. falciparum vaccine candidate proteins. In this study, we have identified and characterized a Pf41 homologue in P. vivax, hence named Pv41, by following such approach and using web-available bioinformatics databases, molecular techniques and immunochemistry assays. Pv41 protein is a 384-amino-acid-long antigen encoded by a single exon that exhibits two $s 48 / 45$ domains characteristic of gametocyte surface proteins. We have also demonstrated Pv41 transcription and expression during late intra-erythrocytic parasite stages and defined its subcellular localization on the parasite surface.
\end{abstract}

(c) 2008 Elsevier Inc. All rights reserved.
Annually, malaria causes the death of nearly 2.5 million people [1] and produces around 350-500 million clinical malaria cases. The 3.2 billion individuals currently estimated to be living at risk of malarial infection, places it as one of the most significant tropical diseases and major public health concerns, being low-income communities the most vulnerable populations [1,2]. Of the four malarial species transmitted to humans, Plasmodium falciparum and Plasmodium vivax have the highest incidences; the former being mainly confined to Africa where it produces the highest mortality rates while the latter has a broader distribution with predominant presence in South Asia, Western Pacific, and South America. Moreover, P. vivax has been recently related to $81 \%$ of cases occurring in Central America and the East Mediterranean [3]. Malaria burden is further aggravated by HIV-malaria coinfection, which has been demonstrated to increase morbidity and mortality rates in areas where both infections are highly prevalent [2].

Over the last decades, the emergence and widespread resistance of the parasite to antimalarial drugs and of its vector (female Anopheles mosquitoes) to insecticides have made it imperative to develop an antimalarial vaccine capable of inducing an efficient protective immune response. Global efforts to pursue such goal have led to the complete sequencing of the $P$. falciparum genome [4] together with its transcriptome [5] and proteome analysis [6,7], which has provided key information for the identification of new drug targets and vaccine candidates. On the contrary, re-

\footnotetext{
* Corresponding author. Address: Molecular Biology Department, Fundación Instituto de Inmunología de Colombia (FIDIC), Carrera 50\#26-20, Bogotá, Colombia. Fax: +57 14815269 .

E-mail address: mapatarr@fidic.org.co (M.A. Patarroyo).
}

search on $P$. vivax has not advanced at the same pace, mainly due to the difficulty of culturing this parasite species in vitro as a consequence of its marked tropism for immature red blood cells (reticulocytes) that account for only $1 \%$ of the total red blood cell count [8]. This difficulty has been largely overcome by the development of bioinformatics tools, which have enabled the identification of potentially antigenic target proteins on the basis of their homology to $P$. falciparum stage-specific vaccine candidates, using as starting point the until recently partially sequenced genome of $P$. vivax released by the Institute for Genomic Research (TIGR) [9-13].

Homology studies have focused mainly on the identification of antigens coating the surface of merozoites (the parasite's bloodstage responsible of invasion to RBCs) and gametocytes (parasite sexual forms) [9,12-14]. The same homology approach has been followed in the study of another protein family known as the Six-cys domain superfamily, whose members include the Pfs230 and Pfs48/45 antigens considered to be important malaria transmission-blocking vaccine candidates due to their expression by parasite sexual stages [15-18]. Six-cys domains have been found to be specific of the genus Plasmodium and consist of six conserved cysteine residues with a characteristic distribution pattern $[19,20]$. A Basic Local Alignment Search Tool (BLAST) screening of the Malaria Genome Sequencing Project sequence databases allowed identifying three new Pf48/45-related genes, which were named $p f 36, p f 41$, and $p f 47$ according to the molecular weight of their deduced mature protein products [20].

Pf41 is a 378 -amino acid-long surface antigen of about $43 \mathrm{kDa}$ encoded by a single exon on chromosome 4 , which has been detected within $P$. falciparum detergent-resistant membrane (DRM)/ lipid-rafts [21]. According to the P. falciparum DRM's transcriptome 
analysis, Pf41 has a signal peptide at its N-terminus, but no transmembrane domains are recognized on its amino acid sequence, neither is it anchored through a glycosylphophatidyl inositol (GPI) tail. Despite not being directly linked to the membrane, Pf41 is coexpressed together with other $12 \mathrm{GPI}$-anchored proteins to which it is non-covalently associated. These proteins have greater expression levels in merozoite stages and lesser in early schizonts. Additionally, Pf41 is strongly recognized by sera from individuals naturally infected by P. falciparum [21], thus confirming its potential as vaccine candidate.

The present study describes the identification and characterization of the Pf41 homologue in P. vivax (Pv41) through the use of molecular biology and immunochemistry techniques.

\section{Materials and methods}

Parasites. The Vivax-Colombia-Guaviare I (VCG-I) P. vivax strain was used as a source of DNA, RNA, and parasite proteins. Parasites were cultured in vivo by successive passes in previously splenectomized Aotus nancymaae monkeys kept at our primate station in Leticia (Amazonas). Late-stage infected RBCs (mainly schizonts) were isolated from 3 to $4 \mathrm{ml}$ blood samples collected from infected animals using a discontinuous Percoll gradient, according to a previously described protocol that allows recovering the largest percentage of infected RBCs [22].

Plasmodium vivax genome sequence source. The partial $P$. vivax genome nucleotide sequence used in this study (Sal-I strain) was retrieved from the TIGR website (http://www.tigr.org/tdb/e2k1/ pva1/).

Parasite DNA. Once parasites had been isolated from $P$. vivax infected-blood samples, DNA was extracted using a Wizard DNA purification system kit (Promega, Wisconsin, USA).

Cloning and sequencing. Plasmodium vivax VCG-I genomic DNA was used as template for PCR amplification. Primers were designed based on the sequence of a putative transcript encoding a Pf41 homologue in $P$. vivax, which was identified by screening the reported $P$. vivax genome sequence using the Pf41 protein sequence as BLAST bait. The so designed primers covered the entire transcript (5'-ATGAAAAGGCTCCTCCTGC-3' forward primer, 5'-CTCCTGGAAGG ACTTGGC-3' reverse primer). PCR products were purified using a Wizard PCR preps kit (Promega, Wisconsin, USA) and cloned into the pEXP5-CT/TOPO vector (Invitrogen, California, USA). Recombinant plasmid DNA was purified using the UltraClean mini plasmid prep purification kit (MO BIO laboratories, Solana Beach, California). Cloned insert integrity was confirmed by sequencing in an automatic ABI PRISM 310 Genetic Analyzer (PE Applied Biosystems, California, USA). For this purpose, two of the clones obtained by independent PCR amplifications were sequenced.

RNA extraction and cDNA synthesis. One microgram of total parasite RNA extracted by the Trizol method and subsequently treated with RQ1 RNase free DNase (Promega, Wisconsin, USA) was used as template for $20 \mu \mathrm{L}$ RT-PCR cDNA synthesis reactions using the SuperScript III enzyme (Invitrogen, California, USA), according to manufacturer's recommendations. In brief, cDNA was synthesized for $60 \mathrm{~min}$ at $50^{\circ} \mathrm{C}$ and then amplified by PCR by the Platinum Pfx DNA polymerase enzyme (Invitrogen, California, USA) for 40 cycles at the following temperatures: $94{ }^{\circ} \mathrm{C}$ for $15 \mathrm{~s}, 54{ }^{\circ} \mathrm{C}$ for $30 \mathrm{~s}, 68^{\circ} \mathrm{C}$ for $60 \mathrm{~s}$ and a final $5 \mathrm{~min}$ extension at $68^{\circ} \mathrm{C}$. An additional PCR was carried out using non-reverse transcribed RNA as template to be used as negative control and thus discard genomic DNA contamination. The RT-PCR products were cloned and sequenced following the same procedure previously described for those obtained from genomic DNA.

Peptide synthesis. Two 20-amino-acid-long peptides were synthesized based on the P. vivax (Sal-I) Pv41 deduced protein se- quence. The sequences in single letter code were: ${ }^{121}$ MEDLKKYLKKRRLTKEKENA ${ }^{140}$ (peptide 35769) and ${ }^{201}$ EDEEELVIEEEQEEEDGEGD ${ }^{20}$ (peptide 35770). Peptides were synthesized by standard solid-phase t-Boc/Bzl peptide synthesis [23] and a glycine and cysteine residues were added at the peptide's $\mathrm{N}$ - and C-terminal ends, respectively, to allow polymerization. The peptides were lyophilized and then characterized by RP-HPLC and MALDI-TOF MS.

Immunization assays in rabbits. Two New Zealand rabbits were subcutaneously inoculated at multiple sites on day 0 with a $1.5 \mathrm{mg}$ mixture of polymerized peptides emulsified in Freund's complete adjuvant (FCA). The same amount of peptide emulsified in Freund's incomplete adjuvant (FIA) was inoculated on days 21 and 42 as booster doses. Sera were collected before the first immunization (pre-immune sera) and 21 days after the third immunization (hyper-immune sera).

Expression and purification of the recombinant protein. The pEXP5-CT/TOPO vector (Invitrogen, California, USA) containing the cloned $p v 41$ gene, adds a six-histidine tag to the protein's C-terminus portion, thereby facilitating protein purification and immune-detection by anti-histidine monoclonal antibodies. The recombinant protein was purified under denaturing conditions using $6 \mathrm{M}$ Urea with a $\mathrm{Ni}^{2+}-\mathrm{NTA}$ affinity resin (Qiagen, California, USA), according to manufacturer's specifications. Protein expression was verified by SDS-PAGE on a $12 \%$ polyacrylamide gel, subsequently stained with Coomassie blue or evaluated by Western blot. The total amount of protein was determined by the bicinchoninic acid assay.

SDS-PAGE and Western blotting. Malarial parasites isolated from P. vivax-infected Aotus monkeys' total blood samples were lysed with a solution containing 20\% SDS, 0.5 M EDTA, $100 \mathrm{mM}$ PMSF, and $100 \mathrm{mM}$ iodoacetamide. Proteins in the lysate were size-separated on a $12 \%$ polyacrylamide gel in the presence of SDS and then electro-transferred to a nitrocellulose membrane. The membrane was blocked with $5 \%$ skimmed milk in PBS-0.05\% Tween, washed thrice with PBS-0.05\% Tween for $5 \mathrm{~min}$ and then cut into strips for individually assessing each rabbit's serum. Strips were incubated with a 1:40 rabbit sera dilution for $1 \mathrm{~h}$ at room temperature, then washed thrice with PBS-0.05\% Tween and subsequently incubated for $1 \mathrm{~h}$ with a 1:4500 secondary antibody dilution (alkaline phosphatase-coupled goat anti-rabbit $\operatorname{IgG}$ ), at room temperature. Antibody excess was removed by washing thrice with PBS-0.05\% Tween before developing the immune reaction with the VIP kit (Vector Laboratories, California, USA), according to manufacturer's instructions.

Indirect immunofluorescence assays. Thin blood smears were made from $P$. vivax-infected blood samples containing mature blood-stage forms (3-5\% parasitemia) on glass slides using a cover-slip. Briefly, slides were first fixed with acetone/methanol, chilled at $4{ }^{\circ} \mathrm{C}$ and then blocked in blocking buffer (PBS-10\% FCS) for $1 \mathrm{~h}$ at $37^{\circ} \mathrm{C}$. Once blocked, slides were incubated with primary antibody (anti-synthetic peptides rabbit polyclonal antibodies diluted 1:10 in blocking buffer) for $1 \mathrm{~h}$ at $37^{\circ} \mathrm{C}$ inside a humid chamber, then washed thrice with PBS and finally incubated with Goat anti-rabbit IgG-fluorescein isothiocyanate conjugate (Sigma, Missouri, USA) diluted 1:40 in blocking buffer. Immunofluorescence was read on an Olympus BX51 fluorescence microscope.

\section{Results and discussion}

Identifying the gene encoding the Six-cys superfamily Pf41 homologous protein in $P$. vivax

A P. vivax homologue to Pf41 was identified by screening the TIGR $P$. vivax genome database with tBLASTn using the Pf41 protein 
sequence (GenBank Accession No. XP001351361) as query. The putative $P$. vivax homologue was located within a 533,304 bp segment on chromosome 3 containing a 1155-pb putative ORF (PlasmoDB gene ID No. PVX_000995) that encodes for a 384 amino acid-long protein with an expected molecular weight of $\sim 44.1 \mathrm{kDa}$.

The PlasmoDB database was also screened searching for orthologous genes to pf41 (PlasmoDB gene ID no. PFD0240c) in other Plasmodium species, finding a similar gene in P. knowlesi and P. yoelii, but not in $P$. berghei or $P$. chabaudi. Only P. knowlesi was considered for the chromosomic comparisons carried out in this study, since previous phylogenetic analysis on Plasmodium have shown a shorter time of divergence between $P$. vivax and $P$. knowlesi than between the former species and $P$. yoelii [24].

The newly identified Pv41 ORF displayed a high BLAST homology score when compared to Pf41, and high identity (Id) (40.7\%) and similarity $(S)(52.4 \%)$ values. An alignment was done between a $P$. falciparum $34 \mathrm{kbp}$, a $P$. vivax $35.51 \mathrm{kbp}$ and a $P$. knowlesi $39 \mathrm{kbp}$ chromosomic regions, in order to confirm gene synteny. The results showed that upstream and downstream ORFs orientation is not only conserved among the three species but that these orthologous ORFs are alike as can be observed by the following Id and $S$ values: $P$. vivax/P. falciparum $\mathrm{Id}=23.5-57.3 \%$ and $S=$ $32-69.5 \%$, P. knowlesi $/ P$. falciparum $\mathrm{Id}=25-58.4 \%$ and $S=33.7-$ $69.1 \%$, and $P$. vivax/P. knowlesi $\mathrm{Id}=51.2-91.6 \%$ and $S=57.7$ 95.1\% (Fig. 1).

According to the sequence analysis, Pv41 (44.1 kDa) is six residues larger than its Pf41 homologue $(43.1 \mathrm{kDa})$ and contains a hydrophobic region towards the N-terminus, possibly corresponding to a signal peptide. This was assessed using SignalP v3.0, finding a cleavage site between amino acids 22 and 23 (AHA-EE) (Fig. 3a), whereas in Pf41 the same cleavage site was located between positions 24 and 25 (VSS-KS). Same as reported for its $P$. falciparum homologue $[20,21]$, no GPI-anchoring sequence was found at the protein's C-terminus when screening Pv41 with the big-PI predictor v3.0 hosted at the ExPASy Molecular Biology Server (http://mendel.imp.ac.at/sat/gpi/gpi_server.html).

The association of Pv41 to the Six-cys superfamily was also corroborated in this study. According to the PlasmoDB and PFAM databases, this protein comprises two sexual stage antigen s48/45 domains (Pfam Accession No. PF07422); one located towards the protein's N-terminal portion between amino acids 22 and 121 and another one towards the C-terminal region between amino acids 247 and 358 (Fig. 3a). The s48/45 domains have a strongly conserved pattern of six cysteines forming three disulfide bonds between them and are characteristic of surface proteins expressed by Plasmodium sexual gametocytes, fulfilling an important and conserved role for parasite fertilization [25,26].

\section{pv41 is transcribed in parasite asexual stages}

Once $P$. vivax RNA was isolated from parasite blood stages, it was treated with DNAase to avoid genomic DNA contamination and subsequently used as template for reverse transcription. The size of the PCR- and RT-PCR amplification products was similar and agreed with the predicted size for pv41 (1152 bp) (Fig. 2). This result indicated that Pv41 is encoded by a single exon, which was later confirmed by sequencing both the genomic and the cDNA amplification products.

When the VCG-I strain parasite sequence was compared with the Sal-I sequence retrieved from the TIGR database, no substitutions were found, suggesting that $p v 41$ is highly conserved since the geographical origin of the P. vivax strains analyzed is totally different. Nevertheless, additional sequences from other $P$. vivax isolates are needed to confirm this assumption.

\section{Pv41 is expressed during parasite asexual stages}

Two 20-amino acid-long peptides were designed based on the deduced $P$. vivax Sal-I Pv41 sequence, choosing peptide fragments lying outside the S48/45 domains that are also present in other Sixcys superfamily proteins, in order to avoid cross-reactivity in immunochemical assays (Fig. 3a). The two synthetic peptides were used as a mixture to immunize two New Zealand rabbits, from which the polyclonal sera used in immunochemical assays to demonstrate Pv41 expression in parasite blood stages were collected.

In the Western blot analysis of the purified recombinant Pv41 protein, a band of around $45.1 \mathrm{kDa}$ was recognized by hyper-immune polyclonal sera raised against the peptide mixture but not by pre-immune sera (Fig. 3b), agreeing with the weight expected for Pv41 according to the initial sequence analysis.

Similarly, when the polyclonal sera were tested against $P$. vivaxlysed parasites isolated from an infected monkey's blood sample, a band at $44.1 \mathrm{kDa}$ was recognized only on membrane stripes that were incubated with hyper-immune sera (Fig. 3c).

\section{Pv41 immunofluorescence pattern}

The localization of the Pv41 protein on the parasite was determined by immunofluorescence assays using P. vivax-infected Aotus

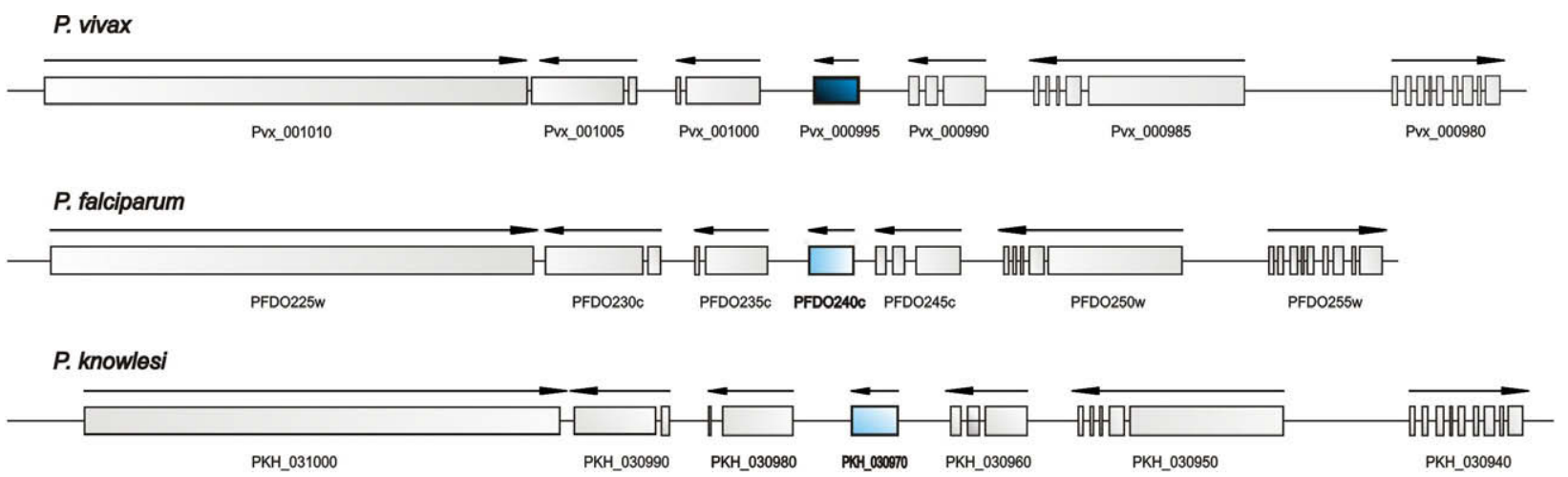

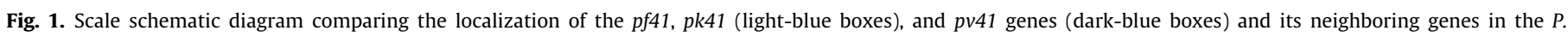

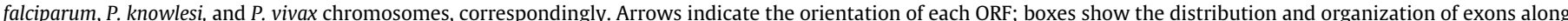

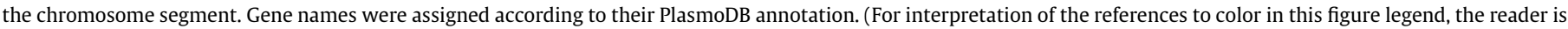
referred to the web version of this article.) 


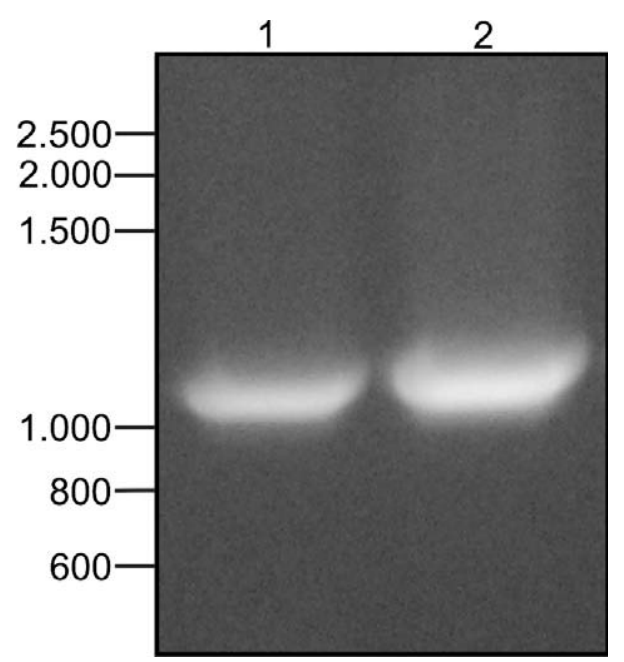

Fig. 2. PCR amplification of the pv41 gene from genomic DNA and cDNA. Lane 1 , segment amplified from genomic DNA by using the $5^{\prime}$ and $3^{\prime}$ primers spanning the entire predicted gene sequence. Lane 2, RT-PCR amplification of total P. vivax DNAse-treated RNA with the same specific primers.

monkey's blood smears and the rabbit 11 hyper-immune serum as primary antibody. The microscopy examination evidenced that Pv41 is dispersed over the merozoite surface during schizont parasite life-cycle stages (Fig. 4). This immunofluorescence pattern agrees with the subcellular localization of Pf41, which is reported to be distributed over the parasite surface on schizont stages; however, in Pv41 we were unable to observe the predominant protein concentration towards the apical pole previously reported for Pf41 on free merozoites [21]. Further confocal microscopy studies could help in deciphering if the localization pattern displayed by this protein on free merozoites is also maintained in $P$. vivax. The fluorescence pattern observed together with the lack of a GPI-anchor suggest that, as occurs in P. falciparum, Pv41 is localized on the par-
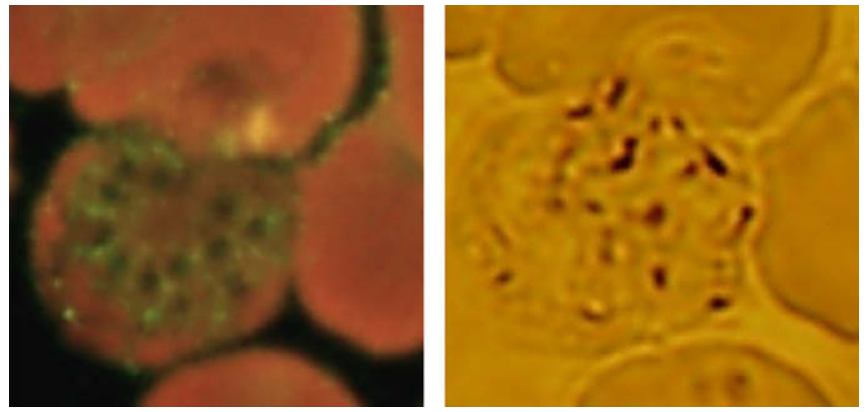

Fig. 4. Cellular localization of Pv41 as determined by immunofluorescence assays in a blood smear using the hyper-immune serum 11 .

asite surface possibly through interactions with GPI-anchored membrane proteins.

In this study, the Pf41 homologue and its encoding gene was identified and characterized in $P$. vivax. The results of this study, together with the structural relationship of Pv41with other transmission-blocking vaccine candidate proteins, its transcription and expression pattern during asexual stages similar to the one described for other proteins implicated in parasite invasion to target cells and its localization on merozoite surface, give support to the inclusion of this antigen as a possible candidate for a multi-stage anti-P. vivax malaria vaccine. Further studies are thus needed in order to elucidate the immunological and protective role of this new antigen.

The homology approach followed in this study allowed identifying this new $P$. vivax antigen based on homology comparisons to $P$. falciparum. However, the recent release of the $P$. vivax complete genome and transcriptome analysis [27,28] will allow a faster identification of new antigens and drug targets, hence strengthening the development of new therapeutic methods against this parasite species.

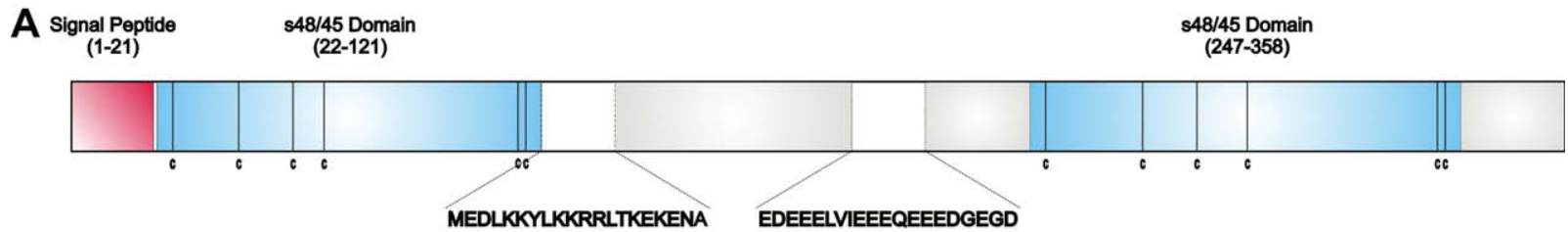

B
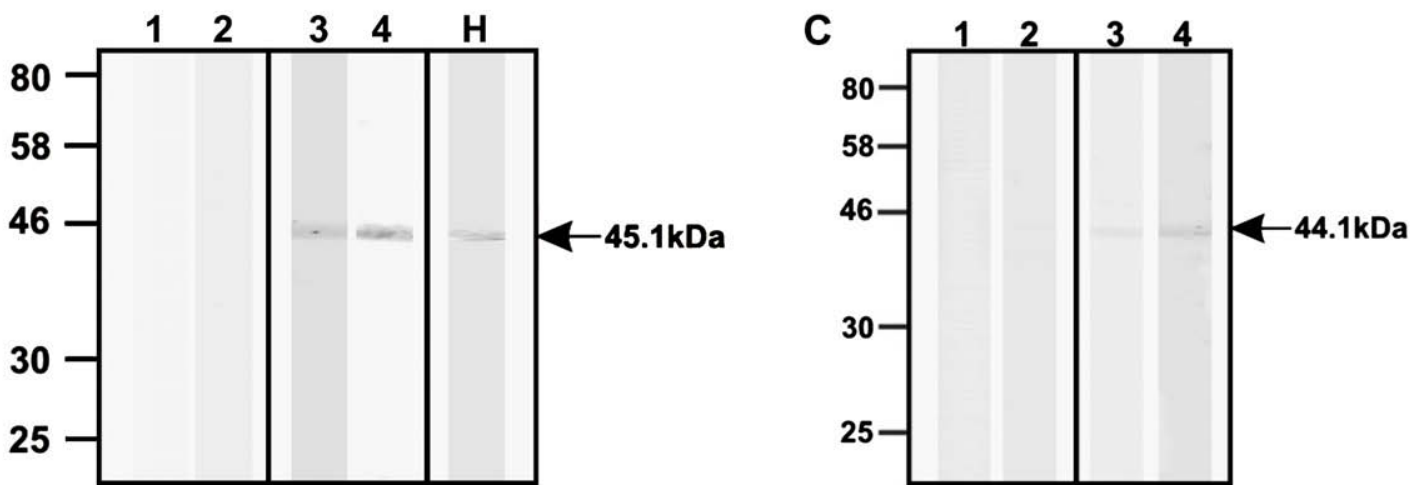

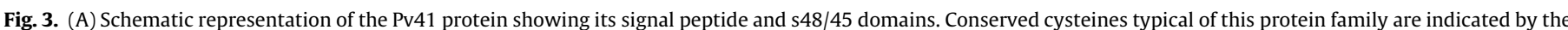

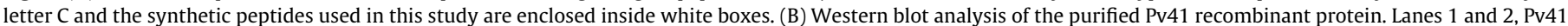

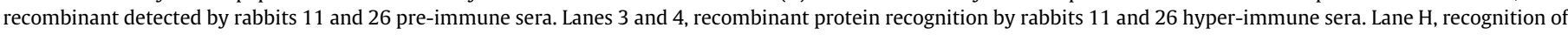

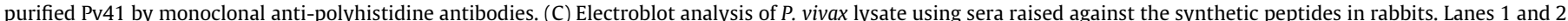

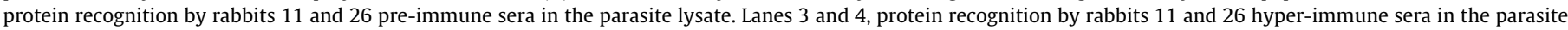
lysate. 


\section{Acknowledgments}

We thank Gina Gallego and Diego Garzón for their technical support. We would also like to thank Nora Martinez for reviewing this manuscript. Special gratitude goes to Professor Manuel Elkin Patarroyo for his invaluable comments and suggestions. This research was supported by President of Colombia's office and the "Instituto Colombiano para el Desarrollo de la Ciencia 'Francisco Jose de Caldas' (COLCIENCIAS)” Contract RC-2008.

\section{References}

[1] A. Waters, Malaria: new vaccines for old?, Cell 124 (2006) 689-693

[2] R. Brierley, Roll Back Malaria issue first global report, Lancet Infect. Dis. 5 (2005) 332-333.

[3] K. Mendis, B.J. Sina, P. Marchesini, R. Carter, The neglected burden of Plasmodium vivax malaria, Am. J. Trop. Med. Hyg. 64 (2001) 97-106.

[4] M.J. Gardner, N. Hall, E. Fung, O. White, M. Berriman, R.W. Hyman, J.M. Carlton, A. Pain, K.E. Nelson, S. Bowman, I.T. Paulsen, K. James, J.A. Eisen, K. Rutherford, S.L. Salzberg, A. Craig, S. Kyes, M.S. Chan, V. Nene, S.J. Shallom, B. Suh, J Peterson, S. Angiuoli, M. Pertea, J. Allen, J. Selengut, D. Haft, M.W. Mather, A.B. Vaidya, D.M. Martin, A.H. Fairlamb, M.J. Fraunholz, D.S. Roos, S.A. Ralph, G.I. McFadden, L.M. Cummings, G.M. Subramanian, C. Mungall, J.C. Venter, D.J Carucci, S.L. Hoffman, C. Newbold, R.W. Davis, C.M. Fraser, B. Barrell, Genome sequence of the human malaria parasite Plasmodium falciparum, Nature 419 (2002) 498-511.

[5] Z. Bozdech, M. Llinas, B.L. Pulliam, E.D. Wong, J. Zhu, J.L. DeRisi, The transcriptome of the intraerythrocytic developmental cycle of Plasmodium falciparum, PLoS Biol. 1 (2003) E5.

[6] L. Florens, M.P. Washburn, J.D. Raine, R.M. Anthony, M. Grainger, J.D. Haynes, J.K. Moch, N. Muster, J.B. Sacci, D.L. Tabb, A.A. Witney, D. Wolters, Y. Wu, M.J. Gardner, A.A. Holder, R.E. Sinden, J.R. Yates, D.J. Carucci, A proteomic view of the Plasmodium falciparum life cycle, Nature 419 (2002) 520-526.

[7] N. Hall, M. Karras, J.D. Raine, J.M. Carlton, T.W. Kooij, M. Berriman, L. Florens, C.S. Janssen, A. Pain, G.K. Christophides, K. James, K. Rutherford, B. Harris, D. Harris, C. Churcher, M.A. Quail, D. Ormond, J. Doggett, H.E. Trueman, J. Mendoza, S.L. Bidwell, M.A. Rajandream, D.J. Carucci, J.R. Yates 3rd, F.C. Kafatos, C.J. Janse, B. Barrell, C.M. Turner, A.P. Waters, R.E. Sinden, A comprehensive survey of the Plasmodium life cycle by genomic, transcriptomic, and proteomic analyses, Science 307 (2005) 82-86.

[8] C.F. Golenda, J. Li, R. Rosenberg, Continuous in vitro propagation of the malaria parasite Plasmodium vivax, Proc. Natl. Acad. Sci. USA 94 (1997) 6786-6791.

[9] O. Perez-Leal, A.Y. Sierra, C.A. Barrero, C. Moncada, P. Martinez, J. Cortes, Y. Lopez, E. Torres, L.M. Salazar, M.A. Patarroyo, Plasmodium vivax merozoite surface protein 8 cloning, expression, and characterisation, Biochem. Biophys. Res. Commun. 324 (2004) 1393-1399.

[10] M.A. Patarroyo, O. Perez-Leal, Y. Lopez, J. Cortes, J. Rojas-Caraballo, A. Gomez, C. Moncada, J. Rosas, M.E. Patarroyo, Identification and characterisation of the Plasmodium vivax rhoptry-associated protein 2, Biochem. Biophys. Res. Commun. 337 (2005) 853-859.

[11] O. Perez-Leal, A. Mongui, J. Cortes, G. Yepes, J. Leiton, M.A. Patarroyo, The Plasmodium vivax rhoptry-associated protein 1, Biochem. Biophys. Res. Commun. 341 (2006) 1053-1058.

[12] O. Perez-Leal, A.Y. Sierra, C.A. Barrero, C. Moncada, P. Martinez, J. Cortes, Y. Lopez, L.M. Salazar, J. Hoebeke, M.A. Patarroyo, Identifying and characterising the Plasmodium falciparum merozoite surface protein 10 Plasmodium vivax homologue, Biochem. Biophys. Res. Commun. 331 (2005) 1178-1184.

[13] A. Mongui, O. Perez-Leal, S.C. Soto, J. Cortes, M.A. Patarroyo, Cloning, expression, and characterisation of a Plasmodium vivax MSP7 family merozoite surface protein, Biochem. Biophys. Res. Commun. 351 (2006) 639-644.

[14] A. Mongui, D.I. Angel, C. Guzman, M. Vanegas, M.A. Patarroyo, Characterisation of the Plasmodium vivax Pv38 antigen, Biochem. Biophys. Res. Commun. (2008).

[15] R. Carter, Transmission blocking malaria vaccines, Vaccine 19 (2001) 23092314.

[16] G. Pradel, Proteins of the malaria parasite sexual stages: expression, function and potential for transmission blocking strategies, Parasitology 134 (2007) 1911-1929.

[17] A. Saul, Mosquito stage, transmission blocking vaccines for malaria, Curr. Opin. Infect. Dis. 20 (2007) 476-481.

[18] K.C. Williamson, Pfs230: from malaria transmission- vaccine candidate toward function, Parasite Immunol. 25 (2003) 351-359.

[19] R. Carter, A. Coulson, S. Bhatti, B.J. Taylor, J.F. Elliott, Predicted disulfidebonded structures for three uniquely related proteins of Plasmodium falciparum, Pfs230, Pfs48/45 and Pf12, Mol. Biochem. Parasitol. 71 (1995) 203-210.

[20] T.J. Templeton, D.C. Kaslow, Identification of additional members define a Plasmodium falciparum gene superfamily which includes Pfs48/45 and Pfs230, Mol. Biochem. Parasitol. 101 (1999) 223-227.

[21] P.R. Sanders, P.R. Gilson, G.T. Cantin, D.C. Greenbaum, T. Nebl, D.J. Carucci, M.J. McConville, L. Schofield, A.N. Hodder, J.R. Yates 3rd, B.S. Crabb, Distinct protein classes including novel merozoite surface antigens in raft-like membranes of Plasmodium falciparum, J. Biol. Chem. 280 (2005) 40169-40176.

[22] P.M. Andrysiak, W.E. Collins, G.H. Campbell, Concentration of Plasmodium ovale- and Plasmodium vivax-infected erythrocytes from nonhuman primate blood using Percoll gradients, Am. J. Trop. Med. Hyg. 35 (1986) 251-254.

[23] R.A. Houghten, General method for the rapid solid-phase synthesis of large numbers of peptides: specificity of antigen-antibody interaction at the level of individual amino acids, Proc. Natl. Acad. Sci. USA 82 (1985) 5131-5135.

[24] A.A. Escalante, E. Barrio, F.J. Ayala, Evolutionary origin of human and primate malarias: evidence from the circumsporozoite protein gene, Mol. Biol. Evol. 12 (1995) 616-626.

[25] M.R. van Dijk, C.J. Janse, J. Thompson, A.P. Waters, J.A. Braks, H.J. Dodemont H.G. Stunnenberg, G.J. van Gemert, R.W. Sauerwein, W. Eling, A central role for P48/45 in malaria parasite male gamete fertility, Cell 104 (2001) 153164.

[26] D.L. Gerloff, A. Creasey, S. Maslau, R. Carter, Structural models for the protein family characterized by gamete surface protein Pfs 230 of Plasmodium falciparum, Proc. Natl. Acad. Sci. USA 102 (2005) 13598-13603.

[27] Z. Bozdech, S. Mok, G. Hu, M. Imwong, A. Jaidee, B. Russell, H. Ginsburg, F. Nosten, N.P. Day, N.J. White, J.M. Carlton, P.R. Preiser, The transcriptome of Plasmodium vivax reveals divergence and diversity of transcriptional regulation in malaria parasites, Proc. Natl. Acad. Sci. USA (2008).

[28] J.M. Carlton, J.H. Adams, J.C. Silva, S.L. Bidwell, H. Lorenzi, E. Caler, J. Crabtree, S.V. Angiuoli, E.F. Merino, P. Amedeo, Q. Cheng, R.M. Coulson, B.S. Crabb, H.A. Del Portillo, K. Essien, T.V. Feldblyum, C. Fernandez-Becerra, P.R. Gilson, A.H. Gueye, X. Guo, S. Kang'a, T.W. Kooij, M. Korsinczky, E.V. Meyer, V. Nene, I. Paulsen, O. White, S.A. Ralph, Q. Ren, T.J. Sargeant, S.L. Salzberg, C.J. Stoeckert, S.A. Sullivan, M.M. Yamamoto, S.L. Hoffman, J.R. Wortman, M.J. Gardner, M.R. Galinski, J.W. Barnwell, C.M. Fraser-Liggett, Comparative genomics of the neglected human malaria parasite Plasmodium vivax, Nature 455 (2008) 757763. 